\title{
A bifurcated plantaris muscle: another confirmation of its high morphological variability? Another type of plantaris muscle
}

\author{
A. Smędra' ${ }^{1}$, Ł. Olewnik², P. Łabętowicz', 2, D. Danowska-Klonowska3 ${ }^{3}$ M. Polguj ${ }^{4}$, J. Berent ${ }^{1}$ \\ ${ }^{1}$ Department of Forensic Medicine, Medical University of Lodz, Poland \\ 2 Department of Anatomical Dissection and Donation, Medical University of Lodz, Poland \\ ${ }^{3}$ Department of Histology and Embryology, Medical University of Lodz, Poland \\ ${ }^{4}$ Department of Normal and Clinical Anatomy, Medical University of Lodz, Poland
}

[Received: 6 August 2020; Accepted: 18 August 2020; Early publication date: 22 August 2020]

\begin{abstract}
The plantaris muscle usually begins with a short, narrow belly in the popliteal fossa at the lateral supracondylar line of the femur and the knee joint capsule. Then it forms a long and slender tendon and usually inserts into the calcaneal tuberosity on the medial side of Achilles tendon. Nevertheless, many anatomical variations of distal attachment have been described. Cases of atypical proximal origin are reported less frequently. In this paper, we have presented a case of a two headed plantaris muscle. First head attached to the condyle of the femoral bone, medially and inferiorly to the lateral head of the gastrocnemius muscle. The second one originated from the popliteal surface of the femur, just above the intercondylar fossa. According to present literature, no such case with atypical proximal origin was presented. Such information has potentially clinical significance during the surgical procedures performed in the area of the popliteal fossa. (Folia Morphol 2021; 80, 3: 739-744)
\end{abstract}

Key words: anatomical variations, plantaris muscle, plantaris tendon, rare variant

\section{INTRODUCTION}

The plantaris muscle (PM) is a small, flat, spindle-shaped muscle located in the posterior region of the knee, slightly above and medially to the lateral head of the gastrocnemius muscle (GM) [16]. The proximal attachment is located on the popliteal surface of the femoral bone above the lateral condyle on the articular capsule [16]. From this origin, the short muscle belly develops into a long, thin tendon, which usually runs in the "space" between the GM and the soleus muscle inserting on to the medial calcaneus and adjacent fibrous tissues [28].
Due to its attachments, it is involved in plantar flexion of the foot at the ankle joint and in knee flexion at the knee joint. In reality, however, it weakly assists the gastrocnemius and soleus muscles to perform these two movements. The PM contains a large number of muscle spindles, so it's considered as a proprioceptive organ for the larger, more powerful flexors of the ankle joint, transferring information about the position of the foot and about movement $[16,28]$.

But still, since neither proprioceptive or flexor functions are affected when plantaris is absent or

Address for correspondence: A. Smędra, MD, PhD, Chair and Department of Forensic Medicine, Medical University of Lodz, ul. Sędziowska 18a, 91-304 Łódź, Poland, tel: +48 508257657, fax: +48 426544293, e-mail: karolanka@wp.pl

This article is available in open access under Creative Common Attribution-Non-Commercial-No Derivatives 4.0 International (CC BY-NC-ND 4.0) license, allowing to download articles and share them with others as long as they credit the authors and the publisher, but without permission to change them in any way or use them commercially. 

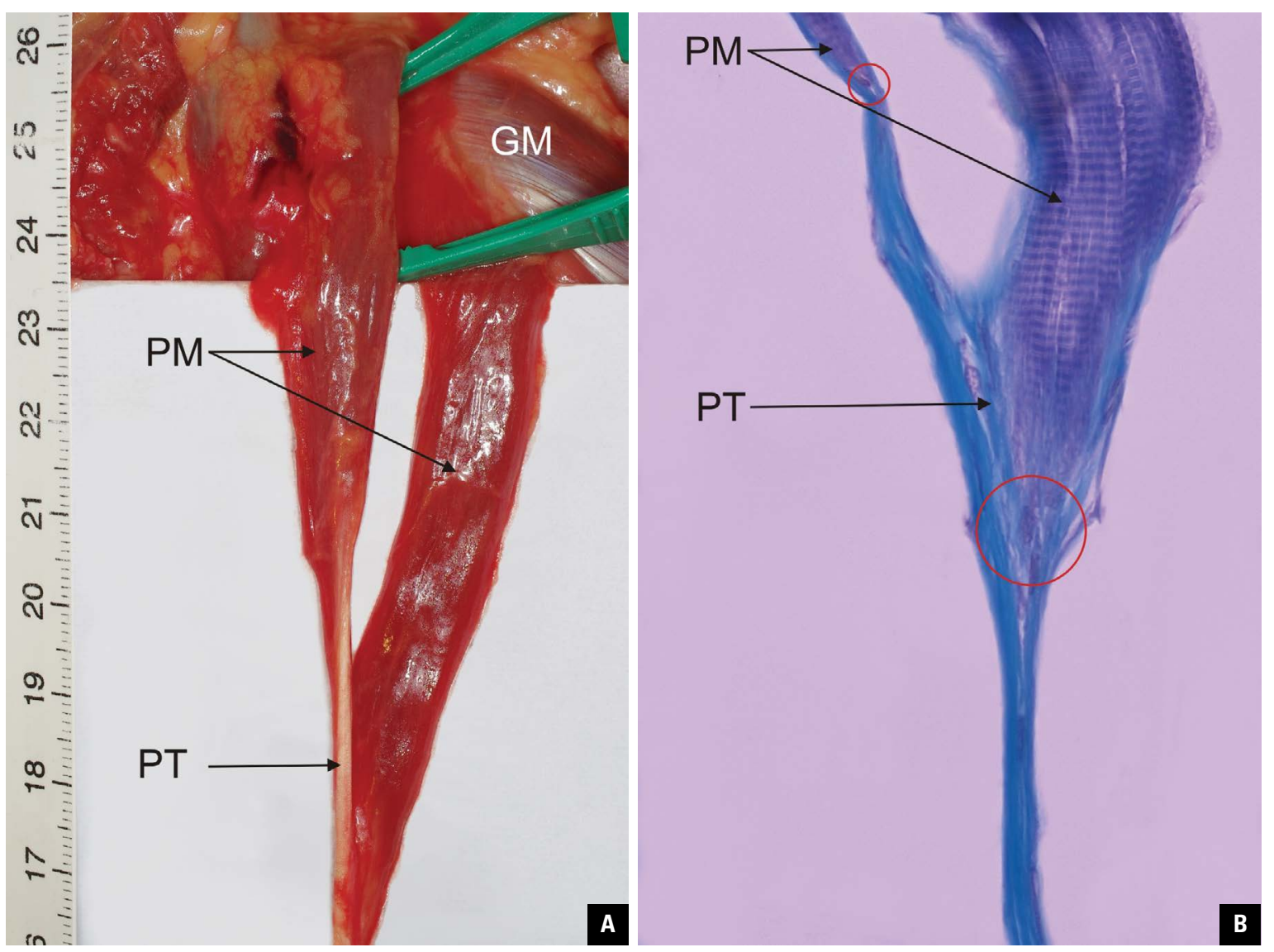

Figure 1. A. Right plantaris muscle with two bellies (PM — plantaris muscle, PT — plantaris tendon); GM — gastrocnemius muscle; B. The plantaris muscle, Mallory staining, mag. $400 \times$. Muscle transitions to the tendon are marked (red circles).

removed, some authors consider this muscle to be vestigial in humans.

It should be noted that PM is not always present. According to different data, this muscle is not present in $4-20 \%$ of subjects $[3,7,21,26]$. Cases of a double PM, unilaterally [11] or bilaterally [10], have also been reported.

Anatomical variability in the morphology of the muscle and its attachments may affect both the function of the lower limb (potential predisposition to Achilles tendon tendinopathy) $[21,30]$ and the surgical procedures, including those involving the use of the plantaris tendon for reconstruction procedures. The tendon of this muscle is long and can be used for the reconstruction of anterior talofibular ligaments, calcaneofibular ligaments and flexor tendons in the lower extremities. Removal of the PM does not typically hinder the patient's lower extremity function in the presence of a normal soleus and gastrocnemius. Injury to the PM either on its own, or in combination with gastrocnemius, or soleus damage, can represent the cause of the clinical condition known as tennis leg [6]. It must be noted that some cases of the use of this tendon for the repair of the atrioventricular valve have also been reported [25].

Findings related to deep venous thrombosis in the calf can be mistaken for those of tennis leg and thus must be kept in mind in the differential diagnosis of clinical findings suggestive of this condition. Other differentials may include a ruptured Baker's cyst, and calf neoplasms [13].

In the present paper, we describe a case of a PM with two completely separate heads, which confirms the introduced classification of origin (rare cases) by Olewnik et al. [22].

\section{CASE REPORT}

In the Chair and Department of Forensic Medicine, a forensic autopsy of a 68-year-old woman was carried out. During the dissection of the muscles, it was found that the right PM had two heads (Fig. 1A). The heads were carefully assessed in situ with respect to their length and width, as were the proximal and distal attachment and the course of the tendon. 
Table 1. Measurement results in millimetres

\begin{tabular}{lcc}
\hline & I & II \\
\hline Belly length & 63.64 & 58.21 \\
Tendon length to the connection point & 8.21 & 2.21 \\
Tendon width at muscle-tendon junction & 1.96 & 1.10 \\
Whole tendon length & 328.21 & 328.21 \\
\hline
\end{tabular}

The first (lateral) head was attached to the lateral condyle of the femur and to the lateral head of the GM, whereas the second (medial) head originated from the femoral popliteal surface, just above the intercondylar fossa. The bellies then passed into a long tendon oriented towards the medial side of the calf. Photographic documentation with a measuring tape was prepared. The muscle was cut and measured using an electronic calliper (Mitutoyo Corporation, Kawasaki-shi, Kanagawa, Japan). Measurement results are given in the Table 1.

The muscle was then fixed in $4 \%$ buffered formalin solution for histological investigations. After fixation, the microscopic specimens stained with haematoxylin and eosin as well as with Mallory's method were prepared. Inspection under the microscope revealed that the heads of the investigated muscle had a normal histological structure (Fig. 1B).

\section{DISCUSSION}

Based on the literature, the PM may be a residual structure, a remnant of the primitive flexor of the toes. According to this assumption, the tuberosity of the calcaneus bone has then become a junction between the two parts of the primary flexor (plantaris tendon and plantaris aponeurosis), which are attached to it but not connected with each other. According to Cruveilhier [4], the change in the position of the foot relative to the remainder of the extremity was the result of the loss of connection between the PM and the plantaris aponeurosis. It is worth noting that the situation is different in the case of the palmaris longus, which has not lost connection with the palmar fascia [23].

The loss of the plantaris tendon connection to the plantaris aponeurosis gave rise to the secondary connection to the calcaneus [4]. In many mammals, including apes, the plantaris is attached to the plantar aponeurosis. In most ruminants and horses; however, the plantaris attaches to the Achilles tendon similar as in humans. A study of the comparative anatomy of mammals reveals no phylogenetically coherent story based on either the size or the location of the attachments of the plantaris. Embryological development in man supports the idea supported by McMurrich that the plantaris is a derivative of the deeper portion of the lateral head of the gastrocnemius. When absent, it is likely that this separation has failed to take place during ontogeny [15].

While the human PM is clearly in a position to flex the knee and to plantarflex the foot at the ankle, its motor functions are obviously trivial. A point often overlooked in the assessment of a muscle's function; however, is that most muscles have a sensory function in addition to their more obvious motor function. While all muscles contain scattered muscle spindles among their more numerous and larger motor fibres, some small muscles have an exceptionally high number of spindles and should be thought of as sensory or proprioceptive organs rather than as motor organs. Often these small sensory muscles are found to be in close association with much larger motor muscles - PM goes together with gastrocnemius and soleus.

The PM is a structure characterized by high anatomic variability. First, it may not be present at all, second, there are many variations in its attachments and third, it can have two or even three bellies (heads) [22].

As it was previously mentioned, according to different data, this muscle is not present in $4-20 \%$ of subjects. Studies usually do not show significant statistical differences in occurrence between sexes and body sides.

The plantaris is exceedingly variable in origin. It may take origin from the inferior extremity of the lateral limb of the linea aspera; the posterior ligament of the knee at the intercondylar space; the fascial covering of the popliteus; the fibula, between the flexor hallucis longus and the peroneus longus; the oblique line of the tibia, under cover of the soleus; the fascia of the leg; the lateral condyle of the femur above the origin of the lateral head of the gastrocnemius and when bicipital, any two of the above mentioned areas [5]. With regard to the differences in proximal attachment, Olewnik et al. [18] described six types of origin and proposed a new classification of plantaris origin. The most common was type I (48.4\%) divided into two subtypes (A-B): subtype $A$, attaching to the lateral head of the GM, lateral femoral condyle and to the capsule of the knee joint, and subtype $B$, attaching to the lateral head of the GM, the lateral 
femoral condyle, knee joint capsule and the popliteal surface of the femur. The second most common type was type II (25\%), attaching to the capsule of the knee joint and, indirectly, to the lateral head of the GM through the lateral femoral condyle. The third most common type was type III (10.15\%), attaching to the lateral femoral condyle and the knee joint capsule. Type IV (6.25\%), the rarest type, attached to the lateral femoral condyle, knee joint capsule and to the iliotibial band. Type $V(8.6 \%)$ originated only from the lateral condyle of the femur. Type VI (1.6\%) was separated for "rare cases" [18].

The distal attachment of the plantaris varies - it usually inserts into the posterosuperior aspect of the calcaneus via the Achilles tendon; however, it can insert into neighbouring structures along it's normal course deep to the gastrocnemius and superficial to the medial aspect of the gastrocnemius and soleus, the flexor retinaculum, the dorsomedial border of the calcaneal tendon near or at its insertion, or distally into the fascia overlying the calcaneus or the plantar aponeurosis $[1,12,17,20,21,24,27,29]$. The specific course of the PT and type of insertion may significantly affect the onset of Achilles midportion tendinopathy. Especially type II (characterised by insertion to the calcaneal tuberosity on the medial side, along with the Achilles tendon of the plantaris tendon which was beaded in common parathendon with the calcaneal tendon) may predispose a patient to this condition [20].

Regarding the cases of bifurcated PM, it has been reported to occur unilaterally [11] and bilaterally [10]. According to Herzog (2011) [8], PM with accessory belly has a prevalence of $6.3 \%$ in 1000 consecutive magnetic resonance imaging exams of the knee. However, cadaveric studies suggest a much lower incidence [10, 24]. In fact, in autopsy study of 750 bodies, Daseler and Anson (1943) [5] did not report the identification of such muscle. When the muscle has two bellies, one of them may have a typical attachment, and the other may be attached in a different area; both bellies can be attached typically or both atypically. Christy and Sathialakshmi [3] described a case in which one of the bellies was attached on the supracondylar line above the lateral condyle, while the other was attached to the oblique popliteal ligament. The popliteal artery was medial to the PM attachment [3]. By contrast Sawant et al. [24] described a rare variation of the muscle with presence of two heads taking origin together from lower part of lateral supracondylar line and oblique popliteal ligament [17]. In the present case, the muscle with two bellies was located unilaterally, as in the publication of Christy and Sathialakshmi (2019) [3]. However, such a case, in which one of the heads is attached to the lateral femoral condyle, medially and below the lateral head of the GM and the other originates on the popliteal surface of the femoral bone just above the intercondylar fossa, has not yet been described. The cases described so far relate to attachments located in other areas. For example, Kotian et al. (2013) [9] described a case in which the muscle had a typical origin on the lateral condyle and then bifurcated into two bellies, one superior and one inferior, whose location differed in relation to the popliteal vessels and the sciatic nerve. The superior head ran in front of these structures, and the inferior head was posterior to them [9]. It should be added that in the case in question, a histological examination was performed, which revealed that there were actually two bellies - two areas of muscle transition to the tendon were clearly visible. Previous publications also did not describe the histological structure of the identified bifurcated PM, which may raise doubts since macroscopic assessment might not always be accurate.

Using the classification prepared by Olewnik et al. 2020 [18], this type should be allocated in type VI - "rare cases". However, it should be emphasized that such type has not yet been described.

According to the authors, the second belly size, location and potential impact on adjacent structures determine how its presence affects the function of the extremity. The presence of the second belly may be mistaken for the presence of a tumour during the examination, so orthopaedists and rehabilitation specialists should be aware that a patient may present such anatomical variation. In the authors' opinion, it would be necessary to consider whether the PM with two bellies may be more prone to disruption. As is known from the literature, plantaris tendon rupture may cause symptoms similar to deep vein thrombosis [14], which can be a significant clinical problem. The second belly may also interfere with the structures located in its vicinity, other muscles, vessels and nerves, especially when muscular hypertrophy occurs. In cases of compression of the vessels or nerves, symptoms suggesting other conditions such as neuropathy, varicose veins or the aforementioned thrombosis may occur. It should be noted that any 
anatomical variability may affect the functioning of the patient. With regard to the differences in proximal attachment, Olewnik et al. [19] described a case in which the PM was attached to the capsule of the knee joint, medially to the lateral head of the crural GM, which resulted in abnormalities in the further course of the muscle.

The presence of a two-headed PM may result in symptoms and disorders that normally do not occur, hinder the diagnostics of the popliteal region and affect the surgical procedures performed in that area (e.g. the tibial nerve, the common fibular nerve, and the popliteal artery and vein). In the authors' opinion, all cases of anatomical variations, not only in the vicinity of the distal attachment but also in that of the initial attachment, should be published, as this may be essential for clinicians, for example, in connection with planned surgical procedures in the popliteal area.

\section{CONCLUSIONS}

The PM is characterised by high morphological variability. The presence of two bellies may potentially affect the surgical procedures performed in the area of the popliteal fossa. Knowledge of anatomical variants of the PM is crucial during surgery in that region. The classification of the PM origin should be extended to include described variant in "type $\mathrm{VI}$ ".

\section{Conflict of interest: None declared}

\section{REFERENCES}

1. Alfredson $\mathrm{H}$. Midportion Achilles tendinosis and the plantaris tendon. Br J Sports Med. 2011; 45(13): 1023-1025, doi: 10.1136/bjsports-2011-090217.

2. Bergman RA, Afifi AK, Miyauchi R. Illustrated encyclopedia of human anatomic variation. 2020. https://www. anatomyatlases.org/AnatomicVariants/AnatomyHP. shtml.

3. Christy JA, Sathialakshmi V. Unilateral two headed plantaris muscle - a rare variation. Int J Anat Var. 2019; 12(1): 015-016.

4. Cruveilhier J. Anatomie descriptive. 1st ed. Bechet Jeune, Paris 1834: 262-263.

5. Daseler EH, Anson BJ. The plantaris muscle: an anatomical study of 750 specimens. J Bone Joint Surg Am. 1943; 25: 822-827.

6. Gilbert TJ, Bullis BR, Griffiths HJ. Tennis calf or tennis leg. Orthopedics. 1996; 19(2): 179, 182, 184, indexed in Pubmed: 8834295.

7. Harvey FJ, Chu G, Harvey PM. Surgical availability of the plantaris tendon. J Hand Surg Am. 1983; 8(3): 243-247, doi: 10.1016/s0363-5023(83)80151-8, indexed in Pubmed: 6875222.
8. Herzog RJ. Accessory plantaris muscle: anatomy and prevalence. HSS J. 2011; 7(1): 52-56, doi: 10.1007/s11420010-9175-y, indexed in Pubmed: 22294958.

9. Kotian SR, Sachin KS, Bhat KMR. Bifurcated plantaris with rare relations to the neurovascular bundle in the popliteal fossa. Anat Sci Int. 2013; 88(4): 239-241, doi: 10.1007/ s12565-013-0184-z, indexed in Pubmed: 23771697.

10. Rana K, Das S, Verma R. Double plantaris muscle: a cadaveric study with clinical importance. Int J Morphol. 2006; 24(3): 495-498, doi: 10.4067/s071795022006000400032 .

11. Kwinter D, Lagrew J, Kretzer J, et al. Unilateral double plantaris muscle: a rare anatomical variation. Int J Morphol. 2010; 28(4): 1097-1099, doi: 10.4067/s071795022010000400018.

12. Le Double AF. Traite des variations du systeme musculaire de l'hommeet de leur signification au point de vue de I' anthropologie zoologique. Tome II. Schleicher frères, Paris 1897.

13. Leekam RN, Agur AM, McKee NH. Using sonography to diagnose injury of plantaris muscles and tendons. Am J Roentgenol. 1999; 172(1): 185-189, doi: 10.2214/ ajr.172.1.9888765, indexed in Pubmed: 9888765.

14. Lopez GJ, Hoffman RS, Davenport M. Plantaris rupture: a mimic of deep venous thrombosis. J Emerg Med. 2011; 40(2): e27-e30, doi: 10.1016/j.jemermed.2007.12.027, indexed in Pubmed: 19150191.

15. McMurrich J. The phylogeny of the crural flexors. Am J Anat. 1905; 4(1): 33-76, doi: 10.1002/aja.1000040104.

16. Moore KL, Agur AMR, Dalley AF. Clinically Oriented Anatomy. 7th ed. Lippincott Williams\&Wilkins, Philadelphia 2013.

17. Nayak SR, Krishnamurthy A, Prabhu LV, et al. Additional tendinous origin and entrapment of the plantaris muscle. Clinics (Sao Paulo). 2009; 64(1): 67-68, doi: 10.1590/ s1807-59322009000100012, indexed in Pubmed: 19142554

18. Olewnik $\measuredangle$, Kurtys K, Gonera B, et al. Proposal for a new classification of plantaris muscle origin and its potential effect on the knee joint. Ann Anat. 2020; 231: 151506, doi: 10.1016/j.aanat.2020.151506, indexed in Pubmed: 32173563.

19. Olewnik $\measuredangle$, Podgórski $M$, Polguj $M$, et al. The plantaris muscle - rare relations to the neurovascular bundle in the popliteal fossa. Folia Morphol. 2018; 77(4): 785-788, doi: 10.5603/FM.a2018.0039, indexed in Pubmed: 29651792.

20. Olewnik $Ł$, Wysiadecki G, Podgórski M, et al. The plantaris muscle tendon and its relationship with the achilles tendinopathy. Biomed Res Int. 2018; 2018: 9623579, doi: 10.1155/2018/9623579, indexed in Pubmed: 29955614.

21. Olewnik $\measuredangle$, Wysiadecki G, Polguj M, et al. Anatomic study suggests that the morphology of the plantaris tendon may be related to Achilles tendonitis. Surg Radiol Anat. 2017; 39(1): 69-75, doi: 10.1007/s00276-016-1682-1, indexed in Pubmed: 27155667.

22. Olewnik $Ł$, Zielinska N, Karauda P, et al. A three-headed plantaris muscle: evidence that the plantaris is not a vestigial muscle? Surg Radiol Anat. 2020; 42(10): 1189-1193, doi: 10.1007/ s00276-020-02478-8, indexed in Pubmed: 32382814.

23. Pękala PA, Kaythampillai L, Skinningsrud B, et al. Anatomical variations of the plantar fascia's origin with respect to 
age and sex-an MRI based study. Clin Anat. 2019; 32(4): 597-602, doi: 10.1002/ca.23342, indexed in Pubmed: 30701591.

24. Sawant SP, Shaikh ST, More RMA. rare variation of plantaris muscle. Int J Biol Med Res. 2012; 3(4): 2437-2440.

25. Shuhaiber JH, Shuhaiber HH. Plantaris tendon graft for atrioventricular valve repair: a novel hypothetical technique. Tex Heart Inst J. 2003; 30(1): 42-44, indexed in Pubmed: 12638670.

26. Simpson SL, Hertzog MS, Barja RH. The plantaris tendon graft: an ultrasound study. J Hand Surg Am. 1991; 16(4): 708-711, doi: 10.1016/0363-5023(91)90198-k, indexed in Pubmed: 1880370.

27. Smith J, Alfredson H, Masci L, et al. Differential plantaris-Achilles tendon motion: a sonographic and cadaveric investigation. PMR. 2017; 9(7): 691-698, doi: 10.1016/j. pmrj.2016.10.013, indexed in Pubmed: 27789336.

28. Spina AA. The plantaris muscle: anatomy, injury, imaging, and treatment. J Can Chiropr Assoc. 2007; 51(3): 158-165, indexed in Pubmed: 17885678.

29. van Sterkenburg MN, Kerkhoffs GM, Kleipool RP, et al. The plantaris tendon and a potential role in mid-portion Achilles tendinopathy: an observational anatomical study. J Anat. 2011; 218(3): 336-341, doi: 10.1111/j.14697580.2011.01335.x, indexed in Pubmed: 21323916.

30. van Sterkenburg MN, Kerkhoffs GM, van Dijk CN. Good outcome after stripping the plantaris tendon in patients with chronic mid-portion Achilles tendinopathy. Knee Surg Sports Traumatol Arthrosc. 2011; 19(8): 1362-1366, doi: 10.1007/ s00167-011-1514-0, indexed in Pubmed: 21541711. 